\title{
The wisdom lost in knowledge: changes in the face of general practice
}

Wendy-Jane Walton MRCGP

When writing my application for the John Fry Bursary I looked at some of the final works of this great researcher, writer and founding father of the Royal College of General Practitioners. Just a year before his death in 1994 Fry had published Common Diseases, their Nature, Presentation and Care; ${ }^{1}$ and two years before that General Practice, the Facts. ${ }^{2}$ How had things changed since then? I concluded that, if he had been alive to produce new editions today, Fry would have had to start almost from scratch; and I cannot think that he would have been happy with the evolution of general practice.

The past dozen years have seen repeated attempts to reform the system of National Health Service management, with a switch from regional, district and family health service authorities, via GP fundholding, to primary care trusts and strategic health authorities. But the biggest change has come from the new General Medical Services contract, implemented from April 2004 after nearly 80\% of GPs voted to accept. This contract allows GPs to shed responsibility for 'out-of-hours' work (nights and weekends) and links their earnings to externally imposed targets. Out-of-hours care will be the responsibility of 'primary care organizations' and the initial triage will be conducted by NHS Direct - a call service in which nurses work to protocols.

Unfortunately, the new system for out-of-hours care is already being found wanting, and there is talk of emergency services being overwhelmed at night. This crisis might have been predicted from our experience in Shropshire before the new contract came into force. When NHS Direct took over calls to the GP cooperative 'Shropdoc' in January 2002 there was a fourfold rise in emergency ambulance calls, a huge influx of patients into emergency departments and a sharp rise in admissions of febrile children to hospital. This failure of NHS Direct was due partly to an unmanageable volume of calls but partly also to the weaknesses of an algorithm-based system; and what it reflected was the fact that GPs had hitherto been providing an efficient and reliable service out of hours. We are expert at assessing risk

Marden Medical Practice, Shrewsbury, UK

Correspondence to: Holm House, Station Road, Pontesbury, Shrewsbury SY5 OQY, UK

E-mail: wendy-jane@doctors.org.uk and advising accordingly, whereas nurses working to protocols are 'safety-netting' all the time. This may sound like an improvement in patient safety, but the opposite is true if other acute services are then stretched beyond capacity. What government should have done was build upon what was working well. Now, as primary care trusts take over responsibility they are finding that the $£ 6000$ each GP will forfeit to give up out-of-hours work will come nowhere near to meeting the cost of contracting services to give cover. In many areas, including my own, no other service providers are available or capable, and the GP cooperative will continue to provide cover but at greater cost. The resources that went into NHS Direct (and where was the evidence for benefit?) would have been better spent on creating a first-class service by attention to the pay and working conditions of GP cooperatives. Under the new scheme, the quality of services under different primary care trusts already looks patchy.

What else has changed since John Fry's day? A good general practitioner still has the old blend of clinical skills, judgment and empathy but an important attribute today is the ability to use information technology in the effort to filter what is useful and relevant. The mass of information that comes through letter box and computer has never been greater; and patients, too, are increasingly perplexed by health information coming by the same routes. Newspapers and magazines are full of health scares, and it does not help that the unfailing rider to these stories is 'If in doubt consult your GP'. Information from the internet ranges from the excellent to the plain dangerous. The past decade has also seen a continuation of demographic changes, with further ageing of the population. A huge proportion of the workload in primary care is the management of chronic disease. With advances in pharmaceutical treatment of ischaemic heart disease and diabetes mellitus, patients live longer but endure more years of chronic ill-health late in life. In some instances, disease seems to follow drugs: the advent of new antidepressants has led to a vast increase in prescribing. It was partly in the hope of dealing better with chronic disease that the Government favoured the new system in the GP contract whereby 'quality points' are awarded for the recording of measurable outcomes-e.g. blood pressure or cholesterol. For some of us, this is the most demoralizing of all the pressures upon us. The quality 
of a consultation, which depends on interaction and trust, cannot properly be judged in such terms. As Onora O'Neill put it in her Reith Lectures, 'Intelligent accountability . . . is not improved by being wholly standardised or relentlessly detailed, and since much that has to be accounted for is not easily measured it cannot be boiled down to a set of stock performance indicators'. Long before the age of information technology, TS Eliot was expressing similar sentiments: 'Where is the wisdom we have lost in knowledge? Where is the knowledge we have lost in information?' For one thing we can be thankful; a key aspect of our work, the care of dying patients and their relatives has been protected by a Gold Standards Framework in Palliative Care that is entirely independent of the contract.

Let me close with an anecdote. An elderly widow I have known for many years had a routine appointment, booked more than a week previously, after a consultation with a nurse in our ischaemic heart disease clinic. She was frail and on medication for hypertension, but I knew from long experience that her most disabling affliction was anxiety, particularly in relation to matters concerning her health. She walked into the consulting room visibly trembling, looking pale and frightened. I thought some major catastrophe must have taken place and sat her down, making attempts to calm her and obtain a coherent history. This is what emerged. In accordance with National Service Framework Guidelines, this lady had been invited to attend the special clinic. Blood results had shown a modestly raised cholesterol, which warranted, initially, dietary advice, according to the evidence. However, this poor lady had no fundamental understanding of the relationship between blood cholesterol levels, blood pressure, diet and risk (as, indeed do very few professionals), and the outcome of her encounter with the nurse was a state of extreme anxiety that had caused her to throw away the box of chocolates her son had given her for Mother's Day and to endure a week of virtual fasting, misery and sleeplessness, and a concomitant rise in her blood pressure to potentially dangerous levels. I spent a disproportionate amount of time trying to mend the damage that our well-intentioned delivery of preventive medicine had occasioned.

Much has been written about the uneasy relationship between evidence-based and narrative-based medicine. I believe that the vast majority of GPs would prefer to continue with caring medicine, unfettered by contractual data-gathering and performance indicators. All, of course, must keep abreast of life-improving and life-saving developments. A dozen years ago John Fry, looking fondly at his old Beckenham practice and beyond, was able to take an optimistic view of primary care in the UK. Today, there is a genuine threat to its quality and human face.

\section{REFERENCES}

1 Fry J, Sandler G. Common Diseases, their Nature, Presentation and Care, 5th edn. Kluwer Academic, 1993

2 Fry J. General Practice: the Facts. Oxford: Radcliffe Medical, 1993 